\title{
Structure and evolution of the magnetochrome domains: no longer alone
}

\section{Pascal Arnoux ${ }^{1,2,3} *$, Marina I. Siponen ${ }^{1,2,3}$, Christopher T. Lefèvre ${ }^{1,2,3}$, Nicolas Ginet ${ }^{1,2,3}$ and David Pignol 1,2,3*}

1 Commissariat à l'énergie Atomique, DSV, IBEB, Lab Bioenerget Cellulaire, Saint-Paul-lez-Durance, France

${ }^{2}$ Centre National de la Recherche Scientifique, UMR Biol Veget and Microbiol Environ, Saint-Paul-lez-Durance, France

${ }^{3}$ Aix-Marseille Université, Saint-Paul-lez-Durance, France

Edited by:

Karim Benzerara, Centre National de la Recherche Scientifique, France

\section{Reviewed by:}

Michael R. Twiss, Clarkson

University, USA

Raz Zarivach, Ben Gurion University

of the Negev, Israel

\section{*Correspondence:}

Pascal Arnoux and David Pignol,

Commissariat à l'énergie Atomique,

DSV, IBEB, Lab Bioenerget

Cellulaire, Saint-Paul-lez-Durance

F-13108, France

e-mail: pascal.arnoux@cea.fr;

david.pignol@cea.fr
Magnetotactic bacteria (MTB) can swim along Earth's magnetic field lines, thanks to the alignment of dedicated cytoplasmic organelles. These organelles, termed magnetosomes, are proteolipidic vesicles filled by a 35-120 nm crystal of either magnetite or greigite. The formation and alignment of magnetosomes are mediated by a group of specific genes, the mam genes, encoding the magnetosome-associated proteins. The whole process of magnetosome biogenesis can be divided into four sequential steps; (i) cytoplasmic membrane invagination, (ii) magnetosomes alignment, (iii) iron crystal nucleation and (iv) species-dependent mineral size and shape control. Since both magnetite and greigite are a mix of iron (III) and iron (II), iron redox state management within the magnetosome vesicle is a key issue. Recently, studies have started pointing out the importance of a MTB-specific c-type cytochrome domain found in several magnetosome-associated proteins (MamE, $\mathrm{P}, \mathrm{T}$, and $\mathrm{X}$ ). This magnetochrome (MCR) domain is almost always found in tandem, and this tandem is either found alone (MamT), in combination with a PDZ domain (MamP), a domain of unknown function (MamX) or with a trypsin combined to one or two PDZ domains (MamE). By taking advantage of new genomic data available on MTB and a recent structural study of MamP, which helped define the MCR domain boundaries, we attempt to retrace the evolutionary history within and between the different MCR-containing proteins. We propose that the observed tandem repeat of MCR is the result of a convergent evolution and attempt to explain why this domain is rarely found alone.

Keywords: magnetotactic bacteria, magnetosome, cytochrome, magnetochrome, evolution, iron

\section{INTRODUCTION}

Some bacteria found in aquatic environments display the singular ability to align passively along Earth's or artificial magnetic field lines while they swim. The genetically controlled biomineralization of magnetic nanocrystals makes this magnetotaxis possible. Made of iron oxide (magnetite, $\mathrm{Fe}^{2+} \mathrm{Fe}_{2}^{3+} \mathrm{O}_{4}$ ) and/or iron sulfide (greigite, $\mathrm{Fe}^{2+} \mathrm{Fe}_{2}^{3+} \mathrm{S}_{4}$ ), these nanomagnets are each embedded in a proteolypidic membrane, forming magnetosomes. These magnetosomes are aligned within the cytoplasm of magnetotactic bacteria (MTB), acting as a compass needle for orientation. A tentative selective advantage would be an efficient localization of the cells in vicinity of the oxic-anoxic transition zone in the water column at their preferred position in the oxygen (and perhaps redox potential) gradient. Since their first scientific description by RP Blakemore in 1975 (Blakemore, 1975), major breakthroughs in MTB isolation and cultivation, combined with advances in genome sequencing technologies have led to ever increasing amounts of information on their ecology, physiology, phylogeny, and evolution (Bazylinski et al., 2013).

Both cultured and uncultured MTB studied thus far are found within the domain Bacteria and affiliated with three phyla: (i) the Proteobacteria phylum with MTB belonging to the Alpha-, Gamma-, and Deltaproteobacteria classes, (ii) the Nitrospirae phylum, including several uncultured strains and, (iii) the Planctomycetes-Verrucomicrobia-Chlamydiae (PVC) (Lefevre and Bazylinski, 2013). Regardless the phylogenetic affiliation, the magnetosomes biomineralized by a given species display a very narrow size range (from about 35 to $120 \mathrm{~nm}$ ) and a given shape (e.g., cubooctahedric, elongated prismatic, bullet shaped). For any given strain, magnetosomes are aligned in chains of constant length and number along the long axis of the cell. When both greigite and magnetite are synthesized, magnetosomes loaded with either mineral are found within the same chain (Lefèvre et al., 2011). Taken together these observations suggest a tight genetic control of the molecular mechanisms governing magnetosome biogenesis. This was confirmed by every comparative genomic analyses published to date with the identification of a series of genes involved in magnetosome biomineralization, specific to and present in MTB, called mam (magnetosome membrane) genes. The mam genes are organized in clusters in the genome of MTB, in some cases defining a bona fide magnetosome genomic island (MAI) (Komeili, 2012). Currently, 13 of these genomic regions have been sequenced, covering all but the PVC phylum of the MTB phylogenetic tree (Grünberg et al., 
2001; Matsunaga et al., 2005; Jogler et al., 2009, 2011; Nakazawa et al., 2009; Schübbe et al., 2009; Lefèvre et al., 2013a,b; Ji et al., 2013). A core gene set composed of mamA, B, I, E, K, M, O, $P$ and $Q$ is conserved among all MTB regardless the chemical composition of the nanocrystal, with an additional gene, mamL, in magnetite-producers. These genes are regrouped in mam $A B$ or mamAB-like operons, referring to the genetic organization described in the paradigm strains Magnetospirillum magneticum AMB-1 and Magnetospirillum gryphiswaldense MSR-1 (Lefevre et al., 2013a). These in silico analyses are nicely confirmed by genetic and biochemical approaches in these 2 strains where the $\operatorname{mam} A B$ operon alone is sufficient for magnetite biomineralization and magnetosomes organization (Murat et al., 2010; Ullrich and Schüler, 2010; Lohsse et al., 2011). Other than bioinformatics predictions, a very limited number of molecular mechanisms have been experimentally evidenced so far. One can cite MamK, a bacterial actin-like protein forming in vitro and in vivo filaments involved in the magnetosome chain assembly (Rioux et al., 2010; Draper et al., 2011; Sonkaria et al., 2012; Ozyamak et al., 2013), MamJ that link the magnetosome to the MamK filament (Scheffel et al., 2006; Scheffel and Schüler, 2007) and MamA that coats the outside of the magnetosome and presumably helps the localization of other magnetosome associated proteins (Zeytuni et al., 2011).

Amongst the Mam proteins, a series of predicted redox proteins exhibit a $c$-type cytochromes motif endemic in MTB and potentially play a role in the iron biocrystallization process that takes place inside the magnetosome (Siponen et al., 2012). The magnetochrome (MCR) domain contains a $\mathrm{CXXCH}$ motif that forms a $c$-type heme-binding site, which is only found in four proteins associated with the magnetosome (MamP, E, T, X, see Table 1 for a list of MCR containing proteins). It is usually present as a tandem repeat, rarely alone or in more repeats, and in all cases the MCR-containing proteins are predicted to be associated to the magnetosome membrane through a single membrane spanning $\alpha$-helix. This original wrapping of $c$-type cytochromes inevitably suggests their participation in an electron transfer chain. Whether it concerns bioenergetics to drive iron import, manage the redox balance of the iron pool or any other molecular mechanisms requiring electron transport is still an open question. Nevertheless, recent studies on MamE, MamX, and MamP were published, hinting at potential functions for MCR domains during magnetosome biogenesis.

In a recent study focused on the biochemistry of MamP and its structural characterization, it was found that MamP displays ferroxidase activity (Siponen et al., 2013). Because of the presence of ferric reductases in MTB (Zhang et al., 2013), as well as the presence of ferrous diffusion facilitators encoded in the MAI (Uebe et al., 2011), $\mathrm{Fe}$ (II) is likely the most readily available form of iron for crystal growth. Since both magnetite and greigite are a mix of iron(III) and iron(II), this implies the presence of Fe(II) oxidation occurring in the magnetosome. MCR-containing proteins such as MamP would be involved in the control of the Fe(II) and $\mathrm{Fe}(\mathrm{III})$ ratio required for magnetite biomineralization (Siponen et al., 2013). This function is supported by in vitro mineralization experiments. Thus, MamP is able to induce magnetite mineralization in the sole presence of $\mathrm{Fe}(\mathrm{II})$, whereas chemical synthesis
Table 1 | list of MCR containing proteins.

\begin{tabular}{|c|c|c|c|c|c|}
\hline Bacteria & MamE & MamP & MamT & MamX & Other \\
\hline AMB-1 & $3^{(*)}$ & 1 & 1 & 1 & \\
\hline MSR-1 & 1 & 1 & 1 & 1 & \\
\hline MS-1 & 2 & 1 & 1 & 1 & \\
\hline MC-1 & 1 & 1 & 1 & 1 & \\
\hline MV-1 & 1 & 1 & 1 & 1 & \\
\hline QH-2 & 1 & 1 & 1 & 1 & \\
\hline SS-5 & $2^{(\S)}$ & 1 & 1 & - & \\
\hline RS-1 $\$$ & $1^{(\dagger)}$ & - & - & - & $\operatorname{MamP}^{*}(\ddagger)$ \\
\hline BW-1 & $1^{(\dagger)}$ & - & - & - & $\operatorname{MamP*}(\ddagger)$ \\
\hline M. bavaricum & $1^{(\mathbf{g})}$ & 1 & (\#) & (\#) & \\
\hline
\end{tabular}

*Three MamE paralogs with small variations: The "classical" MamE (amb0963) with two MCR domains, MamE-Like (amb0410) and LimE or Like-MamE (amb1002) with four MCR domains.

${ }^{\dagger}$ Different from the classical MamE with the PDZ domain replaced by a TauE domain (Trypsin-MCR1-MCR2-TauE).

${ }^{\ddagger}$ MamP* is different from MamP or MamT but contains two putative MCR domains with the following architecture: MCR1-MCR2-PDZ-NitroFeMoCo.

\# Homolog absent but the entire genome has not been sequenced yet.

$\$$ Two paralogs of MamE with one (MamE) containing four MCR domains (MCRA1-MCRA2-Trypsin-MCR1-MCR2-PDZ) and the other (MamE') containing only one MCR domain between the Trypsin and the PDZ domains (TrypsinMCRO-PDZ).

'Contains only one MCR domain (Trypsin-MCRO-PDZ).

requires mixing iron(II) and iron(III) in appropriate proportion (Baumgartner et al., 2013a). MamP ferroxidase activity is then sufficient to produce the iron(III) required for magnetite growth. Siponen et al. observed that the initial formation of the mineral phase is ferrihydrite (an iron(III) oxide), magnetite appearing later in the assay. This suggests that MamP could be involved in ferrihydrite production, an intermediate of magnetite detected in vivo (Baumgartner et al., 2013b; Fdez-Gubieda et al., 2013). Further work using different species is required to firmly establish the role of MamP in vivo, and to determine its electron transfer partner(s).

The redundancy of MCR domains across different proteins of the magnetosome membrane can make their functional characterization somewhat difficult. This is particularly true for the laboratory strain Magnetospirillum magneticum AMB-1, where multiple paralogs of Mam proteins exist. As a consequence, deletion of the MCR domains in one protein might be compensated by the presence of another MCR-containing paralog. This is well illustrated in the study by Quinlan et al. recently published on MamE in this strain (Quinlan et al., 2011). This protein is predicted as a protease belonging to the HtrA/DegP proteases family and is found in every genome of magnetite-producing MTB known to date. Canonical HtrA/DegP proteases possess a trypsin domain followed by two PDZ domains. A variation of this domain organization is found in MamE with the insertion of tandem MCR domains between trypsin and PDZ domains. In M. magneticum AMB-1, the deletion of $\operatorname{limE}$, a paralog of mamE, has no phenotype, but when mamE is also deleted, there is a complete loss of magnetite biomineralization, although empty 
magnetosomes still form chains within the cytoplasm. Transcomplementation of this double mutant with a full mamE restores the wild-type phenotype whereas mamE mutants impaired in the fixation of the two $c$-type hemes only partially complemented the mutant (Quinlan et al., 2011). Complementation with a $m a m E$ variant impaired in its protease activity did not restore the wild-type phenotype. These observations suggest that the MCR tandem in MamE possesses a limited role in magnetite formation and that the protease function of MamE has a dominant function in crystal nucleation initiation. A search for MCR in this strain however reveals that, beside the MamP, T, X, and MamE, two paralogs of MamE are located elsewhere, one in the magnetosome island (Amb1002; named LimE for Like-MamE Quinlan et al., 2011; 63\% identity with MamE), and another one present in a genomic islet that contains homologous mam genes distinct from the magnetosome island (Amb0410, named MamE-like Rioux et al., 2010; 53\% identity with MamE). It is therefore possible that the functions of the MCR domains of MamE are maintained by the other MamE-like proteins, especially if one considers that one of these proteins (Amb0410) is an out-group in the MCRcontaining family of proteins, as it possesses four MCR domains instead of the classical tandem usually found (see below). Further work is needed to clarify the functional roles of the MCR domains of MamE.

The situation is somehow clearer in $M$. gryphiswaldense strain MSR-1 in which only MamE, $\mathrm{P}, \mathrm{T}$, and $\mathrm{X}$ are predicted to possess two MCR domains, with no paralogs inside or outside the magnetosome island. Recently, the role of MamX was investigated in this species (Raschdorf et al., 2013). MamX is associated to the magnetosome membrane and contains a pair of MCR domains. The authors observed the presence of rare wild-type like magnetite crystals flanked by poorly crystalline particles in a $\Delta \operatorname{mam} X$ strain. These "flake-like" particles were identified as hematite (Raschdorf et al., 2013). Both magnetite and hematite particles evolved concomitantly, suggesting that hematite is not an intermediate in magnetite formation and rather that the fate of these individual particles was determined at an early stage. Transcomplementation of the $\Delta m a m X$ strain yielded a WT phenotype whereas complementation with a variant of MamX devoid of the MCR domains did not restore the WT phenotype. Together with mam $Y$ and mam $Z$, mamX belongs to the mamXY operon, which is a signature of magnetotactic Alphaproteobacteria. Its neighbor MamZ contains a predicted ferric reductase domain fused to a transporter belonging to the major facilitator superfamily (MFS). The phenotypes of the mamX, mam $Z$ and mamH mutants led the authors to propose a functional MamXZH interaction that would form an iron oxidoreductase and transport complex through the magnetosome membrane. The understanding of this system and the role of the MCR-containing protein MamX need further study. For example, electron transfer partners and directionality of electron flow remain unknown for MamX.

Altogether the bioinformatics and experimental data available on MCR-containing Mam proteins suggest their involvement in iron redox chemistry to ensure the proper mineralization of magnetosomes. By taking advantage of new genomic data available and recent structural data on MCR domain, we attempt to retrace the evolutionary history of this domain within and between the different MCR-containing proteins. We also hypothesize on the reasons why this domain is rarely found alone but rather in tandem repeats.

\section{RESULTS \\ STRUCTURE OF MCR}

Newly available information on the structure of the MamP protein from the ovoid magnetotactic bacterium MO-1 has laid the groundwork for understanding the structural basis of MCR function (Siponen et al., 2013). Prior to this X-ray structure determination work, the $c$-type cytochrome domains of MamP were already proposed to define a novel domain that is only found in MTB (Siponen et al., 2012). The primary structure suggested that in MamP, a PDZ was followed by two $\mathrm{CX}_{2} \mathrm{CH}$ heme attachment motifs, defining two magnetochrome domains (MCR1 and MCR2). The overall fold of MamP in the crystal revealed a dimer with both monomers mainly stabilized by numerous contacts between their PDZ domains. The first magnetochrome domain (MCR1) is in contact with its own PDZ domain, while the second (MCR2) is projected above the PDZ domain of the other monomer. This structural study allowed the first fold description of a MCR domain, substantiating its uniqueness at the structural level. Indeed, a structural homology search with DaliLite v.3 returns no significant hits, demonstrating the specificity and the uniqueness of these domains (Holm and Park, 2000). Examining the MCR domains in the structure reveals that each MCR clearly defines a single domain, confirming that the MCR is a mono heme $c$-type cytochrome domain and not a diheme as it may have been inferred from its seemingly repeated structure (Figure 1A).

Based on bioinformatic analysis the minimal unit defining the MCR domain can be described as $[\mathrm{P} / \mathrm{T} / \mathrm{H}] \mathrm{HX}_{5-9} \mathrm{CX}_{2} \mathrm{CH}$. A more in-depth structural analysis suggests that the entire MCR domain is composed of 20 amino acids in MamP (see Materials and Methods section). A detailed examination of the structure identified two hydrophobic residues, which delineate the $\mathrm{N}$-terminal and C-terminal regions of the MCR domain. In the fold, these two residues interact hydrophobically to close off the domain. Based on these observations, we proposed a more accurate delineation of a typical MCR domain: $\psi 1 \mathrm{X}_{5-9} \mathrm{PHX}_{5-9} \mathrm{CX}_{2} \mathrm{CH} \psi 2$ (Figure 1B). The MCR domain starts with a hydrophobic residue $(\psi 1)$ directly contacting the heme moiety. This is followed by a $\mathrm{PH}$ motif providing the 6 th heme ligand and located five residues upstream (in MamP) of the $\mathrm{CX}_{2} \mathrm{CH}$ motif anchoring the heme to the polypeptide. Finally, the terminal hydrophobic residue $(\psi 2)$ closes the MCR fold by interacting with the $\psi 1$ residue. Being composed of 19-28 residues, it represents the smallest monoheme cytochrome known to date (the mono-heme cytochrome c-553 from Bacillus pasteurii contains 71 residues surrounding the heme moiety). Overall, this results in a highly solvent-exposed heme moiety, with all four solvent edges exposed (Figure 1C).

As previously mentioned, with the exception of MamT, MCR domains are often found in conjunction with other types of domains. In MamP, the MCR domains are C-terminal to a $\mathrm{PDZ}$ protein-protein interaction domain. The fold observed in the crystal for the entire protein is dimeric showing that the MCRs provide a redox gateway above the crucible formed by the interaction of both PDZ domains (Figure 1A). While structural 
A

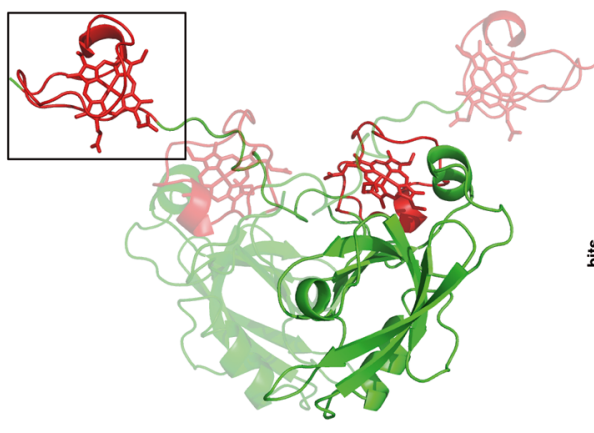

C

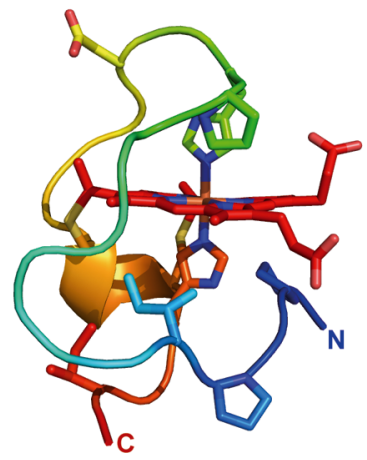

FIGURE 1 | Structure of MCR. (A) Overall structure of the MamP dimer with both monomers colored according to their domain organization (PDZ in green and magnetochrome domains in red), with one monomer rendered in transparency. (B) Weblogo (http://weblogo.berkeley.edu/)
B
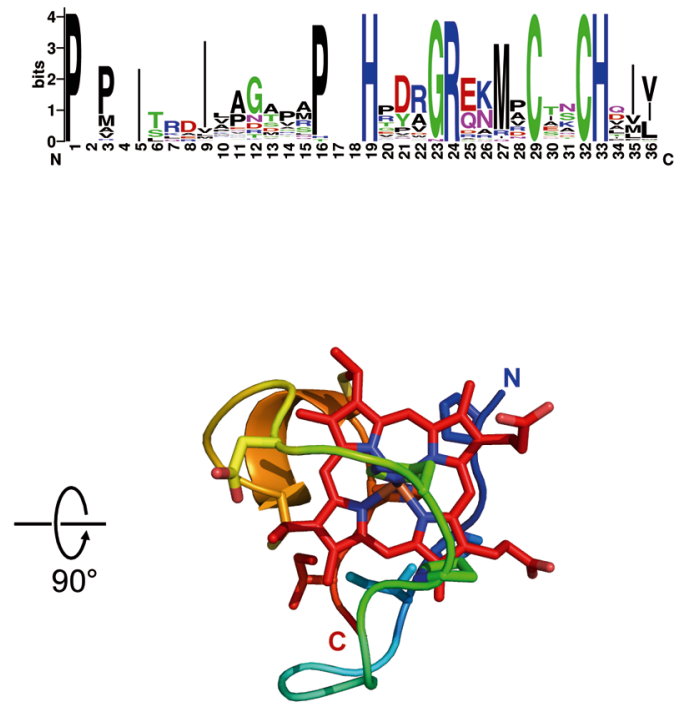

information is still unavailable for the MCR domains of MamX, MamE, and MamT, the fold of the MCR in itself is likely to be very similar. However, it is noteworthy to mention that in the case of MamE, the two MCR domains may adopt a different spatial orientation as that in MamP since there is often a consequent amino acid insertion between both MCRs (30-60 amino acids depending on species). Furthermore, in the case of MamE, the MCR domains are flanked N-terminally by a protease domain and C-terminally by two PDZ domains making it difficult to predict any structural information based on the MamP structure. The MCR domains of MamX could hypothetically form a redox gateway above its domain of unknown function, as seen in MamP, but no substantial evidences exist to support this scenario. Only new structural data on these proteins will allow understanding of the overall organization of MCR within their corresponding proteins.

\section{EVOLUTION OF MCR DOMAINS}

Among the questions about MCR evolution, we are concerned about their occurrence. For example why MCR domains are almost always found in tandem and so rarely alone or repeated more than twice (Table 1)? And for each tandem, are the two repeated MCR domains similar or not? Did they evolve from a single ancestral tandem of MCR domains or rather evolved from independent duplication events? Such intriguing questions can be approached through the evolutionary history of these MCR domains. Because the MCR domain is "endemic" in MTB, tracing back their evolutionary history should be simplified and, as it is representation of a typical magnetochrome domain. (C) Structure of a magnetochrome domain colored from blue (N-Terminus) to red (C-Terminus) and with a few residues conserved in the Weblogo representation shown in stick. found in the core genes set common to all MTB, we are expecting a reasonable diversity in our sample population. The structural studies on MamP described above allow a clear delineation of the domain's boundaries, which should also simplify the constitution of our sample population.

The evolution of a duplicated domain can be considered in two simple evolutionary models where internal duplication of the original domain takes place either before (Figure 2, Model \#1) or after (Figure 2, Model \#2) functional and sequence divergence of the entire protein. In the first case, MCR1 and MCR2 domains would appear as two separate branches in a phylogenetic tree whatever the protein considered (MamE, P, T, X), whereas in the second case the separation would initially occur between the proteins containing the MCR domains forming separate branches for (MamE, P, T, X). At first sight, the first model seems the simplest to explain the functional diversity observed in MCR-containing proteins. Indeed, an initial (and presumably rare) event of internal domain duplication would have taken place, followed by a functional divergence of the proteins. The second model is probably less intuitive as it depicts a single MCR divergence before the duplication events; however this model does not explain why the domain is rarely found alone but almost always in duplicate, unless we think about convergent evolution. An alternative model explaining why the MCR1 and MCR2 domains share more sequence identity within a family would be that there is a evolutionary constraint on the MCR1 and MCR2 that must be kept similar to each other for the dimer to be functional. 


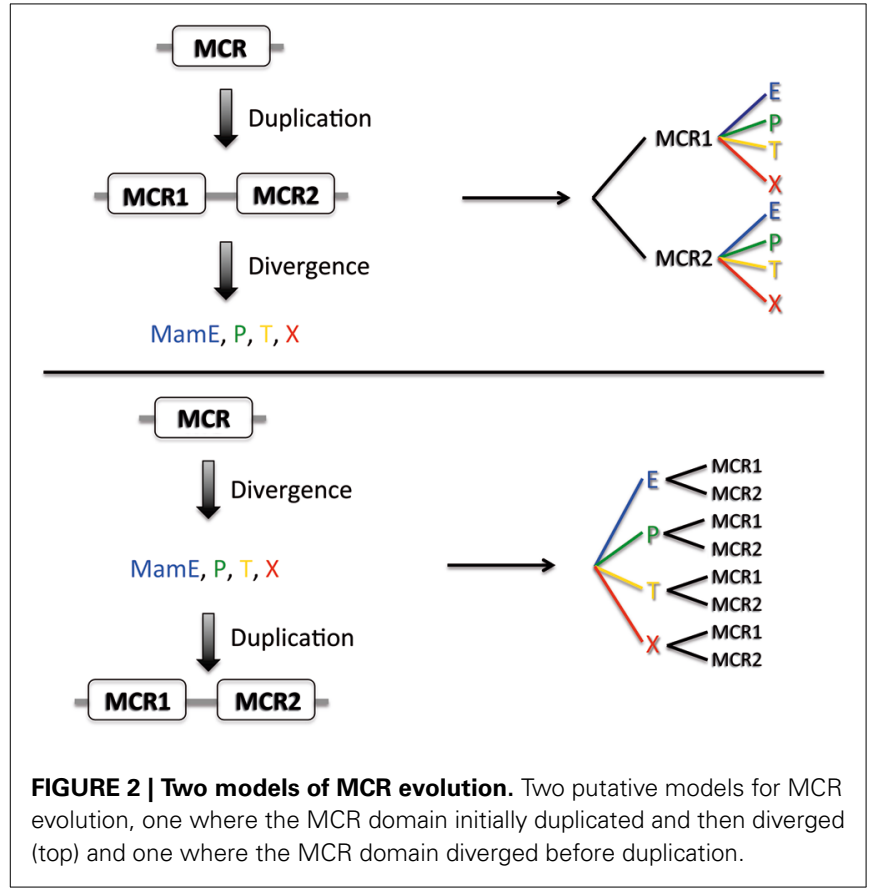

To generate our dataset, we gathered sequences of MCR containing proteins MamE, P, T, and X from 10 species (see Materials and Methods) and separated them into MCR1 and MCR2 as described above. A protein sequences alignment was computed with all the individual MCR using the Muscle algorithm (Edgar, 2004) and a phylogenetic trees built with MEGA5 software (Tamura et al., 2011); the MCR alignment as well as the individual protein sequence alignment are provided as Supplementary Figure 1. The resulting tree presented in Figure 3 displays several branches with clear boundaries, which is already surprising considering the short size of the MCR domains (19 amino acids) and the low bootstrap values when generated (data not shown). Much to our surprise, we found that the MCR domains do not clusterize according to their position in the amino acid sequence (MCR1 or MCR2) as expected for model \#1 but rather form a cluster with the Mam protein they belong to, as predicted in model \#2. For instance in the case of MamE and MamT, the MCR domains, regardless of their numbering, form distinct leaves for each protein. Then within each leaf we observe distinct branches leading to the MCR1 and MCR2. This topology is clearly reminiscent to model \#2 where divergence of the original MCR domain occurred before the internal duplication.

\section{DISCUSSION}

A rather simple evolutionary scheme can be proposed for the MCR-containing Mam proteins where the basic scheme is provided by model \#2: an initial sequence divergence event followed by domain duplication. It is interesting to note that even based on short MCR domain sequences, one can relatively easily infer the nature of the protein to which it belongs (MamE, $\mathrm{P}, \mathrm{T}$ or X).

Whether it is an ancient or more recent evolution, whether or not it is part of the minimal gene set required for magnetosome biosynthesis, the major trend for the magnetochrome domain

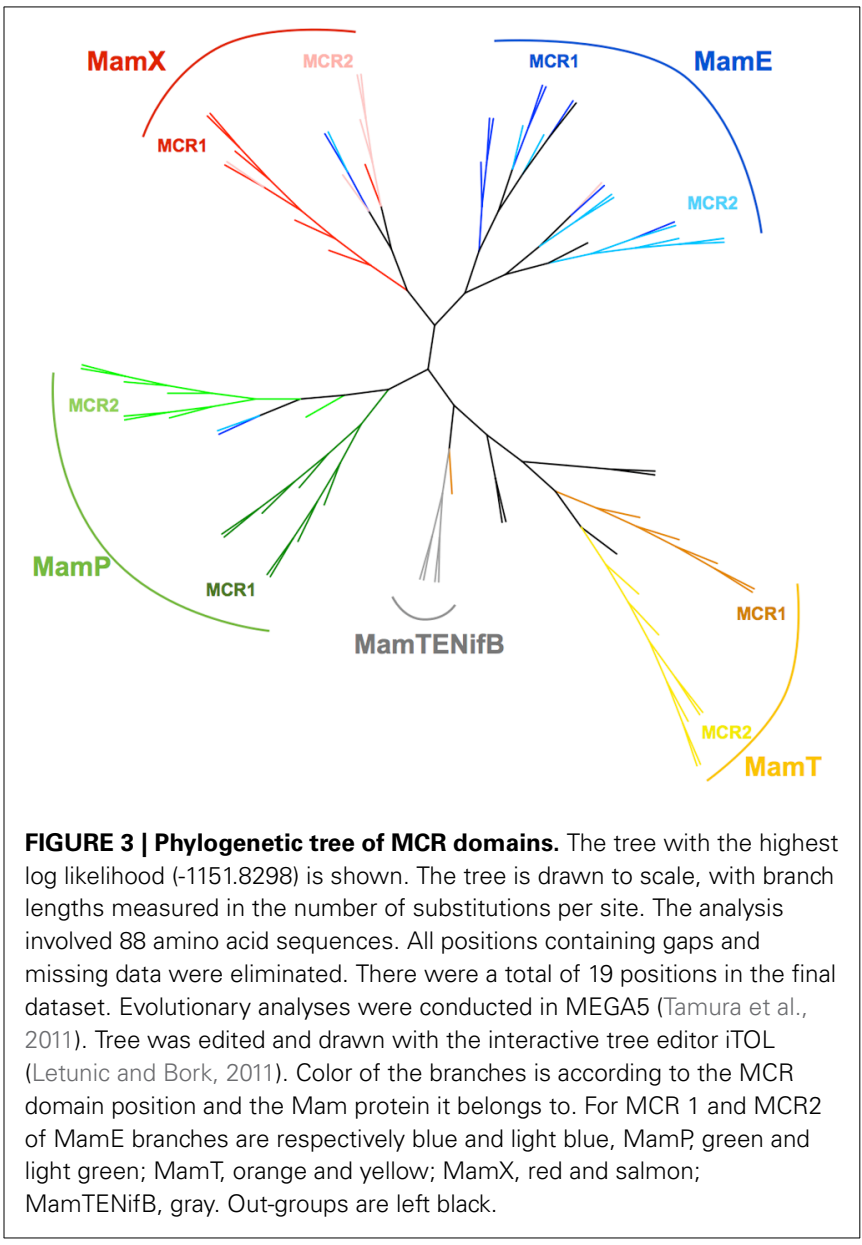

evolution is a tandem duplication after a sequence divergence. It seems that when a new protein with a tandem MCR domain is selected by evolution, it always evolves from a lone MCR domain and not from an existing tandem repeat. For example, it is known that MamX is only present in MTB from the Alphaproteobacteria, suggesting that it evolved relatively recently. However, its evolutionary history based on the MCR domain only suggests that it did not emerge from the tandem of another MCR-containing protein like previously existing MamP or MamE. What we may be witnessing here is an example of convergent evolution where the tandem repeat is linked to the functional role of the protein. Indeed, this domain is almost always found in tandem and there are only rare examples where it is found either, alone (MamE of Candidatus Magnetobacterium bavaricum and MamE' of SS-5) or in triplicate (MamE of strain MC-1). It is tempting to link this pattern to iron and magnetite (or greigite) chemistry. Both magnetite and greigite are a mix of one iron(II) and two iron(III) equivalents. The possibility to abstract or give two electrons by a pair of magnetochrome domains suggests its involvement directly in magnetite or greigite crystal production, not just the iron chemistry that requires a single electron. Such use of two monoheme cytochromes was also suggested to evolve in order to adapt to the storage of the two electron generated from sulfite oxidation (Robin et al., 2013). Although this hypothesis of two 
MCR domains adaptation to a two-electron transfer reaction is tempting, it will be difficult to test and further work is definitely needed in order to better understand the complex connections between MTB and MCR.

A hypothesis concerning the evolution of magnetotactic bacteria suggests they all evolved from a common ancestor 2.5-3.0 billion years ago when levels of atmospheric oxygen were low and anaerobic to microaerobic environments dominated. At this time, magnetite/greigite crystals likely did not serve a role in magnetotaxis but rather in scavenging reactive oxygen species and later, when atmospheric oxygen levels increased, served to aid MTB in navigation (Lefèvre et al., 2013a). This ancestor probably had only the mamAB operon (including mamE and mamP) and the magnetite/greigite crystals have diversified since then. Because magnetochrome-containing proteins are the only redox proteins associated to the magnetosome this raises interesting possibilities possibly linking magnetite/greigite crystal shape to the evolution of these magnetochrome-containing proteins.

The three dimensional structure of MamP showed that the first magnetochrome domain contributed to the formation of a crucible in which iron could be stabilized (Siponen et al., 2013). This structural study enabled a better analysis of the evolutionary history of MCR domains by defining the boundaries of this domain. The structure of other MCR-containing proteins such as MamE or MamX will allow to better define the role of the magnetochrome domain in the context of magnetotaxis. Furthermore, these studies will also allow more robust structurebased sequence alignments. Finally, interesting questions that need to be answered in the future relate to the interaction between these MCR containing proteins and the identification of their electron-transfer partners.

\section{METHODS}

The complete genomes or contigs discussed in this paper include: Magnetospirillum magneticum AMB-1 (GenBank: NC_007626.1), Magnetospirillum gryphiswaldense MSR-1 (GenBank: CU459003.1), Magnetospirillum magnetotacticum MS-1 (JGI project 402922), Magnetococcussp. MC-1 (GenBank: CP000471), Magnetovibrio blakemorei MV-1 (GenBank: FP102531), Magnetospira sp. QH-2 (EMBL: FO538765), strain SS-5 (MamP: JX628772, MamE: JX628767), Candidatus Magnetobacterium bavaricum (Jogler et al., 2011), Desulfovibrio magneticus RS-1 (NC_012795-NC_012797), Candidatus Desulfamplus magnetomortis BW-1 (GenBank: JN830627-JN830646 and JN845570-JN845575).

From a structure based sequence alignment we devised a protein pattern that harvests almost all the magnetochrome domains without many false positive. This pattern is the following:

$$
\begin{aligned}
& {[\mathrm{P}]-\mathrm{x}(0,6)-[\text { IVMLQA }]-\mathrm{x}(6)-[\mathrm{PTH}]-\mathrm{X}(0,2)-[\mathrm{H}]} \\
& -\mathrm{x}(1,3)-[\mathrm{GN}]-\mathrm{x}(1,5)-\mathrm{C}-\mathrm{x}-\mathrm{x}-\mathrm{C}-\mathrm{H}-\mathrm{x}-[\mathrm{IVMLFY}]
\end{aligned}
$$

and much of the false positive belong to a single protein subunit, $\mathrm{NrfB}$ from a formate dependent Cytochrome $c$ nitrite reductase.

Initial multiple alignment were generated using the MUSCLE program (Edgar, 2004), applying the default settings. Alignment were visualizes using Jalview package. Evolutionary trees were obtained using the MEGA5 package (Tamura et al., 2011) and by using the Maximum Likelihood method based on the JTT matrixbased model (Jones et al., 1992). Initial tree(s) for the heuristic search were obtained automatically by applying Neighbor-Join and BioNJ algorithms to a matrix of pairwise distances estimated using a JTT model, and then selecting the topology with superior log likelihood value.

\section{SUPPLEMENTARY MATERIAL}

The Supplementary Material for this article can be found online at: http://www.frontiersin.org/journal/10.3389/fmicb.2014.001 17/abstract

Supplementary Figure 1/ Sequence alignment of MCR domains used in this analysis. Sequence alignment is colored as defined by clustalx in Jalview 2 (Waterhouse et al., 2009).

\section{REFERENCES}

Baumgartner, J., Dey, A., Bomans, P. H. H., Le Coadou, C., Fratzl, P., Sommerdijk, N. A., et al. (2013a). Nucleation and growth of magnetite from solution. Nat. Mater. 12, 310-314. doi: 10.1038/nmat3558

Baumgartner, J., Morin, G., Menguy, N., Gonzalez, T. P., Widdrat, M., Cosmidis, J., et al. (2013b). Magnetotactic bacteria form magnetite from a phosphate-rich ferric hydroxide via nanometric ferric (oxyhydr)oxide intermediates. Proc. Natl. Acad. Sci. U.S.A. 110, 14883-14888. doi: 10.1073/pnas.1307119110

Bazylinski, P. D. A., Lefèvre, C. T., and Schüler, D. (2013). "Magnetotactic bacteria," in The Prokaryotes, eds E. Rosenberg, E. F. DeLong, S. Lory, E. Stackebrandt, and F. Thompson (Berlin; Heidelberg: Springer), 453-494. Available online at: http://link.springer.com/referenceworkentry/10.1007/978-3-642-30141-474 (Accessed October 18, 2013).

Blakemore, R. (1975). Magnetotactic bacteria. Science 190, 377-379. doi: 10.1126/science.170679

Draper, O., Byrne, M. E., Li, Z., Keyhani, S., Barrozo, J. C., Jensen, G., et al. (2011). MamK, a bacterial actin, forms dynamic filaments in vivo that are regulated by the acidic proteins MamJ and LimJ. Mol. Microbiol. 82, 342-354. doi: 10.1111/j.1365-2958.2011.07815.x

Edgar, R. C. (2004). MUSCLE: multiple sequence alignment with high accuracy and high throughput. Nucleic Acids Res. 32, 1792-1797. doi: 10.1093/nar/gkh340

Fdez-Gubieda, M. L., Muela, A., Alonso, J., García-Prieto, A., Olivi, L., FernándezPacheco, R., et al. (2013). Magnetite biomineralization in Magnetospirillum gryphiswaldense: time-resolved magnetic and structural studies. ACS Nano 7, 3297-3305. doi: 10.1021/nn3059983

Grünberg, K., Wawer, C., Tebo, B. M., and Schüler, D. (2001). A large gene cluster encoding several magnetosome proteins is conserved in different species of magnetotactic bacteria. Appl. Environ. Microbiol. 67, 4573-4582. doi: 10.1128/AEM.67.10.4573-4582.2001

Holm, L., and Park, J. (2000). DaliLite workbench for protein structure comparison. Bioinformatics 16, 566-567. doi: 10.1093/bioinformatics/16.6.566

Ji, B., Zhang, S.-D., Arnoux, P., Rouy, Z., Alberto, F., Philippe, N., et al. (2013). Comparative genomic analysis provides insights into the evolution and niche adaptation of marine Magnetospira sp. QH-2 strain. Environ. Microbiol. 16, 525-544. doi: 10.1111/1462-2920.12180

Jogler, C., Kube, M., Schübbe, S., Ullrich, S., Teeling, H., Bazylinski, D. A., et al. (2009). Comparative analysis of magnetosome gene clusters in magnetotactic bacteria provides further evidence for horizontal gene transfer. Environ. Microbiol. 11, 1267-1277. doi: 10.1111/j.1462-2920.2009.01854.x

Jogler, C., Wanner, G., Kolinko, S., Niebler, M., Amann, R., Petersen, N., et al. (2011). Conservation of proteobacterial magnetosome genes and structures in an uncultivated member of the deep-branching Nitrospira phylum. Proc. Natl. Acad. Sci. U.S.A. 108, 1134-1139. doi: 10.1073/pnas.1012694108

Jones, D. T., Taylor, W. R., and Thornton, J. M. (1992). The rapid generation of mutation data matrices from protein sequences. Comput. Appl. Biosci. 8, 275-282.

Komeili, A. (2012). Molecular mechanisms of compartmentalization and biomineralization in magnetotactic bacteria. FEMS Microbiol. Rev. 36, 232-255. doi: 10.1111/j.1574-6976.2011.00315.x 
Lefêvre, C. T., and Bazylinski, D. A. (2013). Ecology, diversity, and evolution of magnetotactic bacteria. Microbiol. Mol. Biol. Rev. 77, 497-526. doi: 10.1128/MMBR.00021-13

Lefèvre, C. T., Menguy, N., Abreu, F., Lins, U., Pósfai, M., Prozorov, T., et al. (2011). A cultured greigite-producing magnetotactic bacterium in a novel group of sulfate-reducing bacteria. Science 334, 1720-1723. doi: 10.1126/science.1212596

Lefèvre, C. T., Trubitsyn, D., Abreu, F., Kolinko, S., De Almeida, L. G. P., De Vasconcelos, A. T., et al. (2013a). Monophyletic origin of magnetotaxis and the first magnetosomes. Environ. Microbiol. 15, 2267-2274. doi: 10.1111/14622920.12097

Lefèvre, C. T., Trubitsyn, D., Abreu, F., Kolinko, S., Jogler, C., De Almeida, L. G., et al. (2013b). Comparative genomic analysis of magnetotactic bacteria from the Deltaproteobacteria provides new insights into magnetite and greigite magnetosome genes required for magnetotaxis. Environ. Microbiol. 15, 2712-2735. doi: 10.1111/1462-2920.12128

Letunic, I., and Bork, P. (2011). Interactive Tree Of Life v2: online annotation and display of phylogenetic trees made easy. Nucleic Acids Res. 39, W475-W478. doi: 10.1093/nar/gkr201

Lohsse, A., Ullrich, S., Katzmann, E., Borg, S., Wanner, G., Richter, M., et al. (2011). Functional analysis of the magnetosome island in Magnetospirillum gryphiswaldense: the mam $\mathrm{AB}$ operon is sufficient for magnetite biomineralization. PLoS ONE 6:e25561. doi: 10.1371/journal.pone.0025561

Matsunaga, T., Okamura, Y., Fukuda, Y., Wahyudi, A. T., Murase, Y., and Takeyama, H. (2005). Complete genome sequence of the facultative anaerobic magnetotactic bacterium Magnetospirillum sp. strain AMB-1. DNA Res. 12, 157-166. doi: 10.1093/dnares/dsi002

Murat, D., Quinlan, A., Vali, H., and Komeili, A. (2010). Comprehensive genetic dissection of the magnetosome gene island reveals the step-wise assembly of a prokaryotic organelle. Proc. Natl. Acad. Sci. U.S.A. 107, 5593-5598. doi: 10.1073/pnas.0914439107

Nakazawa, H., Arakaki, A., Narita-Yamada, S., Yashiro, I., Jinno, K., Aoki, N., et al. (2009). Whole genome sequence of Desulfovibrio magneticus strain RS1 revealed common gene clusters in magnetotactic bacteria. Genome Res. 19, 1801-1808. doi: 10.1101/gr.088906.108

Ozyamak, E., Kollman, J., Agard, D. A., and Komeili, A. (2013). The bacterial actin MamK: in vitro assembly behavior and filament architecture. J. Biol. Chem. 288, 4265-4277. doi: 10.1074/jbc.M112.417030

Quinlan, A., Murat, D., Vali, H., and Komeili, A. (2011). The HtrA/DegP family protease MamE is a bifunctional protein with roles in magnetosome protein localization and magnetite biomineralization. Mol. Microbiol. 80, 1075-1087. doi: 10.1111/j.1365-2958.2011.07631.x

Raschdorf, O., Müller, F. D., Pósfai, M., Plitzko, J. M., and Schüler, D. (2013). The magnetosome proteins MamX, MamZ and MamH are involved in redox control of magnetite biomineralization in Magnetospirillum gryphiswaldense. Mol. Microbiol. 89, 872-886. doi: 10.1111/mmi.12317

Rioux, J.-B., Philippe, N., Pereira, S., Pignol, D., Wu, L.-F., and Ginet, N. (2010). A second actin-like MamK protein in Magnetospirillum magneticum AMB-1 encoded outside the genomic magnetosome island. PLoS ONE 5:e9151. doi: 10.1371/journal.pone.0009151

Robin, S., Arese, M., Forte, E., Sarti, P., Kolaj-Robin, O., Giuffrè, A., et al. (2013). Functional Dissection of the Multi-Domain Di-Heme Cytochrome c550 from Thermus thermophilus. PLoS ONE 8:e55129. doi: 10.1371/journal.pone.0055129

Scheffel, A., Gruska, M., Faivre, D., Linaroudis, A., Plitzko, J. M., and Schüler, D. (2006). An acidic protein aligns magnetosomes along a filamentous structure in magnetotactic bacteria. Nature 440, 110-114. doi: 10.1038/nature04382

Scheffel, A., and Schüler, D. (2007). The acidic repetitive domain of the Magnetospirillum gryphiswaldense MamJ protein displays hypervariability but is not required for magnetosome chain assembly. J. Bacteriol. 189, 6437-6446. doi: 10.1128/JB.00421-07

Schübbe, S., Williams, T. J., Xie, G., Kiss, H. E., Brettin, T. S., Martinez, D., et al. (2009). Complete genome sequence of the chemolithoautotrophic marine magnetotactic coccus strain MC-1. Appl. Environ. Microbiol. 75, 4835-4852. doi: 10.1128/AEM.02874-08

Siponen, M. I., Adryanczyk, G., Ginet, N., Arnoux, P., and Pignol, D. (2012). Magnetochrome: a c-type cytochrome domain specific to magnetotatic bacteria. Biochem. Soc. Trans. 40, 1319-1323. doi: 10.1042/BST20120104

Siponen, M. I., Legrand, P., Widdrat, M., Jones, S. R., Zhang, W.-J., Chang, M. C. Y., et al. (2013). Structural insight into magnetochrome-mediated magnetite biomineralization. Nature 502, 681-684. doi: 10.1038/nature12573

Sonkaria, S., Fuentes, G., Verma, C., Narang, R., Khare, V., Fischer, A., et al. (2012). Insight into the assembly properties and functional organisation of the magnetotactic bacterial actin-like homolog, MamK. PLoS ONE 7:e34189. doi: 10.1371/journal.pone.0034189

Tamura, K., Peterson, D., Peterson, N., Stecher, G., Nei, M., and Kumar, S. (2011). MEGA5: molecular evolutionary genetics analysis using maximum likelihood, evolutionary distance, and maximum parsimony methods. Mol. Biol. Evol. 28, 2731-2739. doi: 10.1093/molbev/msr121

Uebe, R., Junge, K., Henn, V., Poxleitner, G., Katzmann, E., Plitzko, J. M., et al. (2011). The cation diffusion facilitator proteins MamB and MamM of Magnetospirillum gryphiswaldense have distinct and complex functions, and are involved in magnetite biomineralization and magnetosome membrane assembly. Mol. Microbiol. 82, 818-835. doi: 10.1111/j.1365-2958.2011.07863.x

Ullrich, S., and Schüler, D. (2010). Cre-lox-based method for generation of large deletions within the genomic magnetosome island of Magnetospirillum gryphiswaldense. Appl. Environ. Microbiol. 76, 2439-2444. doi: 10.1128/AEM.02805-09

Waterhouse, A. M., Procter, J. B., Martin, D. M. A., Clamp, M., and Barton, G. J. (2009). Jalview version 2 - a multiple sequence alignment editor and analysis workbench. Bioinformatics 25, 1189-1191. doi: 10.1093/bioinformatics/btp033

Zeytuni, N., Ozyamak, E., Ben-Harush, K., Davidov, G., Levin, M., Gat, Y., et al. (2011). Self-recognition mechanism of MamA, a magnetosome-associated TPR-containing protein, promotes complex assembly. Proc. Natl. Acad. Sci. U.S.A. 108, E480-487. doi: 10.1073/pnas. 1103367108

Zhang, C., Meng, X., Li, N., Wang, W., Sun, Y., Jiang, W., et al. (2013). Two bifunctional enzymes with ferric reduction ability play complementary roles during magnetosome synthesis in Magnetospirillum gryphiswaldense MSR-1. J. Bacteriol. 195, 876-885. doi: 10.1128/JB.01750-12

Conflict of Interest Statement: The authors declare that the research was conducted in the absence of any commercial or financial relationships that could be construed as a potential conflict of interest.

Received: 03 December 2013; accepted: 08 March 2014; published online: 25 March 2014.

Citation: Arnoux P, Siponen MI, Lefevre CT, Ginet N and Pignol D (2014) Structure and evolution of the magnetochrome domains: no longer alone. Front. Microbiol. 5:117. doi: 10.3389/fmicb.2014.00117

This article was submitted to Aquatic Microbiology, a section of the journal Frontiers in Microbiology.

Copyright (c) 2014 Arnoux, Siponen, Lefevre, Ginet and Pignol. This is an openaccess article distributed under the terms of the Creative Commons Attribution License (CC BY). The use, distribution or reproduction in other forums is permitted, provided the original author(s) or licensor are credited and that the original publication in this journal is cited, in accordance with accepted academic practice. No use, distribution or reproduction is permitted which does not comply with these terms. 